\title{
On Hilbert's Inequality for Double Series and Its Applications
}

\author{
Zhou Yu and Gao Mingzhe \\ Department of Mathematics and Computer Science, Normal College of Jishou University, Jishou, \\ Hunan 416000, China
}

Correspondence should be addressed to Gao Mingzhe, mingzhegao@163.com

Received 2 September 2007; Accepted 26 March 2008

Recommended by Feng Qi

This study shows that a refinement of the Hilbert inequality for double series can be established by introducing a real function $u(x)$ and a parameter $\lambda$. In particular, some sharp results of the classical Hilbert inequality are obtained by means of a sharpening of the Cauchy inequality. As applications, some refinements of both the Fejer-Riesz inequality and Hardy inequality in $H_{p}$ function are given.

Copyright (C) $2008 \mathrm{Z}$. Yu and G. Mingzhe. This is an open access article distributed under the Creative Commons Attribution License, which permits unrestricted use, distribution, and reproduction in any medium, provided the original work is properly cited.

\section{Introduction}

Let $\left\{a_{n}\right\}$ and $\left\{b_{n}\right\}$ be two sequences of complex numbers. If $\lambda=0,1$, then

$$
\begin{array}{r}
\left|\sum_{m=1-\lambda}^{\infty} \sum_{n=1-\lambda}^{\infty} \frac{a_{m} \bar{b}_{n}}{m+n+\lambda}\right| \leq \pi\left(\sum_{n=1-\lambda}^{\infty}\left|a_{n}\right|^{2}\right)^{1 / 2}\left(\sum_{n=1-\lambda}^{\infty}\left|b_{n}\right|^{2}\right)^{1 / 2}, \\
\left|\sum_{m=1-\lambda}^{\infty} \sum_{\substack{n=1-\lambda \\
m \neq n}}^{\infty} \frac{a_{m} \bar{b}_{n}}{m-n}\right| \leq \pi\left(\sum_{m=1-\lambda}^{\infty}\left|a_{n}\right|^{2}\right)^{1 / 2}\left(\sum_{n=1-\lambda}^{\infty}\left|b_{n}\right|^{2}\right)^{1 / 2},
\end{array}
$$

where the constant factor $\pi$ is the best possible. It is all known that the inequalities (1.1) and (1.2) are called the Hilbert theorem for double series. The two forms (1.1) and (1.2) on the Hilbert inequality were combined into one similar form in some papers (e.g., [1, 2], etc.), 
that is,

$$
\left|\sum_{m=1-\lambda}^{\infty} \sum_{n=1-\lambda}^{\infty} \frac{a_{m} b_{n}}{m+n+\lambda}\right|^{2}+\left|\sum_{\substack { m=1-\lambda \\
\begin{subarray}{c}{n=1-\lambda \\
m \neq n{ m = 1 - \lambda \\
\begin{subarray} { c } { n = 1 - \lambda \\
m \neq n } }\end{subarray}}^{\infty} \frac{a_{m} b_{n}}{m-n}\right|^{2} \leq \pi^{2} \sum_{n=1-\lambda}^{\infty}\left|a_{n}\right|^{2} \sum_{n=1-\lambda}^{\infty}\left|b_{n}\right|^{2} .
$$

Recently, the various extensions on (1.1) appeared in some papers (e.g., [3, 4], etc.).They focalize on changing the denominator of the function of the left-hand side of (1.1). Such as the denominator $(m+n+\lambda)$ is replaced by $\left(\alpha_{m}+\beta_{n}\right)^{\mu}$ in [3], and the denominator $(m+n+\lambda)$ is replaced by $(m u(m)+n v(n))^{\mu}$ in [4]. Some new results in these papers were yielded. The inequality (1.3) is obviously a significant refinement of (1.1) and (1.2). However, both extensions and refinements on (1.2) and (1.3) do not commonly appear in previous papers. The main purpose of the present paper is to establish both an extension and a significant refinement on (1.3). Explicitly, let $u(x)>0 x \in[0,+\infty)$ be a real function and let $\lim _{x \rightarrow \infty} u(x)=+\infty$. If the denominator $(m+n+\lambda)$ of the first term of the left-hand side of (1.3) is replaced by $u(m)+u(n)$, and the denominator $(m-n)$ of the second term of the left-hand side of (1.3) is replaced by $u(m)-u(n)$, then a new inequality established is significant in theory and applications; and as applications, we will give both extensions and refinements on Fejer-Riesz's inequality and Hardy's inequality. For convenience, we introduce some notations and functions as follows:

$$
\begin{aligned}
V(a, b) & =\sum_{m=0}^{\infty} \sum_{\substack{n=0 \\
u(m) \neq v(n)}}^{\infty} \frac{a_{m} \overline{b_{n}}}{u(m)-u(n)}, \\
U_{k}(a, b) & =\sum_{m=0}^{\infty} \sum_{n=0}^{\infty} \frac{a_{m} \overline{b_{n}}}{(u(m)+u(n))^{k}}, \quad k=1,2, \\
\|x\|^{2} & =\sum_{n=0}^{\infty}\left|x_{n}\right|^{2}, \\
T_{k}(x) & =\sum_{n=0}^{\infty} \frac{\left|x_{n}\right|}{u^{k}(n)}, \quad k=1,2, \\
(f, g) & =\int_{0}^{2 \pi} f(t) \overline{g(t)} d t, \\
\|\alpha\|^{2} & =\int_{0}^{2 \pi}|\alpha|^{2} d t, \quad \text { where } \alpha=f, g, h .
\end{aligned}
$$

In particular, when $b=\bar{a}$, the notations $U_{k}(a, a)$ and $V(a, a)$ are denoted, respectively, by $U_{k}(a)$ and $V(a)$. Throughout this paper, we will frequently use these notations, and we stipulate that $Z$ denotes integer set and that $u(n)=Z_{n}+\lambda / 2$, where $Z_{n} \in Z, n \in N_{0}, \lambda$ is an integer or $0<\lambda<1$.

\section{Lemmas}

In order to prove our assertions, we need the following lemmas. 
Lemma 2.1. If both $\sum_{n=0}^{\infty} a_{n}$ and $\sum_{n=0}^{\infty} b_{n}$ are absolute convergent, then

(i) $\sum_{m=0}^{\infty} \sum_{n=0}^{\infty} a_{m} b_{n}$ is absolute convergent,

(ii) $\|a\|^{2}$ and $\|b\|^{2}$ are convergent.

The proof of it has been given in the paper [2]. Hence, it is omitted here.

Lemma 2.2. Let $f, g, h \in L^{2}[0,2 \pi]$, and let $h$ be a variable unit-vector. Then,

$$
|(f, g)|^{2} \leq\|f\|^{2}\|g\|^{2}\left(1-\left(\frac{|(f, h)|}{\|f\|}-\frac{|(g, h)|}{\|g\|}\right)^{2}\right) .
$$

In particular, when $h$ is orthogonal to $f$, we have

$$
|(f, g)|^{2} \leq\|f\|^{2}\|g\|^{2}\left(1-\left(\frac{|(g, h)|}{\|g\|}\right)^{2}\right)
$$

and the equality in (2.2) holds if and only if $f, g$ and $h$ are linearly dependent.

The proof of these results has been given in $[5,6]$.

Define a function by

$$
r(x)=\pi U_{1}(x) \sin 2 \lambda \pi-U_{2}(x) \sin ^{2} \lambda \pi .
$$

Lemma 2.3. Let $\left\{a_{n}\right\}$ and $\left\{b_{n}\right\}$ be two sequences of complex numbers. If $\lambda$ is an integer or $1 / 2 \leq \lambda<1$, then

$$
r(a) r(b) \geq 0
$$

Proof. When $\lambda$ is an integer, it is clear that $r(a) r(b)=0$. So we consider only the case for $1 / 2 \leq$ $\lambda<1$. It is easy to deduce that

$$
\begin{aligned}
& U_{1}(x)=\int_{0}^{1}\left|\sum_{m=0}^{\infty} x_{m} t^{u(m)-1 / 2}\right|^{2} d t>0, \\
& U_{2}(x)=\int_{0}^{1} \frac{d s}{s} \int_{0}^{s}\left|\sum_{n=0}^{\infty} x_{n} t^{u(n)-1 / 2}\right|^{2} d t>0 .
\end{aligned}
$$

When $1 / 2 \leq \lambda<1$, it follows from (2.3) that $r(x)<0$ for any $x \in C$. Hence, we have $r(a) r(b)>0$.

Lemma 2.4. Let $f(z)=\sum_{n=0}^{\infty} a_{n} z^{u(n)-1 / 2}$. If $f(z)$ is analytic in the unit-disc $|z|<1$, then

$$
\left.\left|\frac{1}{2 \pi} \int_{-\pi}^{\pi} t\right| f\left(-e^{i t}\right)\right|^{2} d t|=| V(a) \mid
$$


Proof.

$$
\begin{aligned}
& \left.\left|\int_{-\pi}^{\pi} t\right| f\left(-e^{i t}\right)\right|^{2} d t \mid \\
& =\left|\int_{-\pi}^{\pi} t f\left(-e^{i t}\right) \overline{f\left(-e^{i t}\right)} d t\right| \\
& =\left|\int_{-\pi}^{\pi} t \sum_{m=0}^{\infty} \sum_{n=0}^{\infty} a_{m} \overline{a_{n}}(\cos (\pi+t)+i \sin (\pi+t))^{u(m)-1 / 2}(\cos (\pi-t)+i \sin (\pi-t))^{u(n)-1 / 2} d t\right| \\
& =2 \pi\left|\left(\sum_{m=0}^{\infty} \sum_{\substack{n=0 \\
u(m) \neq u(n)}}^{\infty} \frac{a_{m} \overline{a_{n}}}{u(m)-u(n)}\right) \sin \lambda \pi+i\left(\sum_{\substack{m=0 \\
u(m) \neq u(n)}}^{\infty} \sum_{\substack{n=0 \\
u(m)-u(n)}}^{\infty} \frac{a_{m} \overline{a_{n}}}{u(m)}\right) \cos \lambda \pi\right| \\
& =2 \pi|V(a)| \text {. }
\end{aligned}
$$

Thereby, the relation (2.6) holds.

\section{Theorems and their corollaries}

In order to prove our assertions, we need also to introduce the following functions:

$$
\begin{aligned}
& s_{1}(x)=\frac{\sqrt{2}\left|T_{1}(x) \cos \lambda \pi-(1 / 2 \pi) T_{2}(x) \sin \lambda \pi\right|}{\left(\pi^{2}\|x\|^{2}-r(x)\right)^{1 / 2}}, \\
& s_{2}(x)=\frac{\sqrt{2}\left|T_{1}(x) \sin \lambda \pi-(1 / \pi) T_{2}(x) \sin ^{2}(\lambda \pi / 2)\right|}{\left(\pi^{2}\|x\|^{2}+r(x)\right)^{1 / 2}} .
\end{aligned}
$$

Theorem 3.1. Let $r(x)$ be a function defined by (2.3), let $\left\{a_{n}\right\}$ and $\left\{b_{n}\right\}$ be two nonzero sequences of complex numbers, and let both $\sum_{n=0}^{\infty} a_{n}$ and $\sum_{n=0}^{\infty} b_{n}$ be absolute convergent. Then,

(i) if $\lambda$ is an integer, then

$$
\left|U_{1}(a, b)\right|^{2}+|V(a, b)|^{2} \leq\left(\pi^{2}\|a\|^{2}\|b\|^{2}\right)\left(1-R^{2}\right),
$$

where

$$
R^{2}= \begin{cases}\frac{1}{\pi^{2}}\left\{\left(\frac{T_{1}(a)}{\|a\|}-\frac{T_{2}(b)}{\pi\|b\|}\right)^{2}+\left(\frac{T_{1}(b)}{\|b\|}-\frac{T_{2}(a)}{\pi\|a\|}\right)^{2}\right\} & \text { if } \lambda \text { is odd } \\ \frac{1}{\pi^{2}}\left(\left(\frac{T_{1}(a)}{\|a\|}\right)^{2}+\left(\frac{T_{1}(b)}{\|b\|}\right)^{2}\right) & \text { if } \lambda \text { is even; }\end{cases}
$$

(ii) if $0<\lambda<1$, then

$$
\left|U_{1}(a, b) \cos 2 \lambda \pi-\frac{1}{2 \pi} U_{2}(a, b) \sin 2 \lambda \pi\right|^{2}+|V(a, b)|^{2} \leq\left\{\pi^{2}\|a\|^{2}\|b\|^{2}-\frac{1}{\pi^{2}} r(a) r(b)\right\}\left(1-R^{2}\right),
$$


where $R^{2}=\min \left\{\left(s_{1}(a)-s_{2}(b)\right)^{2},\left(s_{1}(b)-s_{2}(a)\right)^{2}\right\}, s_{i}(x)(i=1,2)$ is defined by (3.1). In particular, when $1 / 2 \leq \lambda<1$, we have $r(a) r(b)>0$.

Proof. Define two functions by

$$
\begin{aligned}
& f(a, t)=\sum_{m=0}^{\infty} a_{m} \sqrt{t} \sin (u(m)) t \\
& g(b, t)=\sum_{n=0}^{\infty} b_{n} \sqrt{t} \cos (u(n)) t, \quad t \in[0,2 \pi] .
\end{aligned}
$$

Since both $\sum_{n=0}^{\infty} a_{n}$ and $\sum_{n=0}^{\infty} b_{n}$ are absolute convergent by Lemma 2.1, the double series $\sum_{m=0}^{\infty} \sum_{n=0}^{\infty} a_{m} b_{n}$ is absolute convergent. Accordingly, $f(a, t) g(b, t)$ is uniformly convergent in the interval $[0,2 \pi]$. Thereby, the interchange in order of summation and integration can be made. In what follows, we stipulate that the interchanges in order of summation and integration are justified. It is easy to deduce that

$$
\begin{aligned}
\|f\|^{2} & =\pi^{2}\|a\|^{2}-r(a), \\
\|g\|^{2} & =\pi^{2}\|b\|^{2}+r(b), \\
|(f, g)| & =\left|\int_{0}^{2 \pi} f(a, t) \overline{g(b, t)} d t\right| \\
& =\pi\left|V(a, b)+\left(U_{1}(a, b) \cos 2 \lambda \pi-\frac{1}{2 \pi} U_{2}(a, b) \sin 2 \lambda \pi\right)\right|,
\end{aligned}
$$

where $r(x)$ is a function defined by (2.3). By Lemma 2.2, we have

$$
\begin{aligned}
& \left|V(a, b)+\left(U_{1}(a, b) \cos 2 \lambda \pi-\frac{1}{2 \pi} U_{2}(a, b) \sin 2 \lambda \pi\right)\right|^{2} \\
& \quad=\frac{1}{\pi^{2}}|(f, g)|^{2} \leq \frac{1}{\pi^{2}}\|f\|^{2}\|g\|^{2}(1-r) \\
& \quad=\frac{1}{\pi^{2}}\left\{\pi^{2}\|a\|^{2}-r(a)\right\}\left\{\pi^{2}\|b\|^{2}+r(b)\right\}(1-r),
\end{aligned}
$$

where $r=(|(f, h)| /\|f\|-|(g, h)| /\|g\|)^{2}, h$ is a variable unit-vector, it can be properly chosen in accordance with our requirement.

(i) When $\lambda$ is an integer, it is known from (2.3) that $r(x)=0$.

We select $h_{1}=\sqrt{2 t} / 2 \pi$ it is easy to deduce that $\left\|h_{1}\right\|=1$, and

$$
\left|\left(f, h_{1}\right)\right|=\left|\sqrt{2}\left(\sum_{m=0}^{\infty} \frac{a_{m}}{u(m)}\right)\right| .
$$

Since the series $\sum_{n=0}^{\infty} a_{n}$ is absolute convergent, it is justified that the complex number $a_{n}$ is replaced by $\left|a_{n}\right|$ in (3.8). Hence, we have

$$
\left|\left(f, h_{1}\right)\right|=\sqrt{2} T_{1}(a) .
$$


Similarly

$$
\left|\left(g, h_{1}\right)\right|= \begin{cases}\frac{\sqrt{2}}{\pi} T_{2}(b) & \text { if } \lambda \text { is odd } \\ 0 & \text { if } \lambda \text { is even. }\end{cases}
$$

We therefore obtain that

$$
r_{1}=\left(\frac{\left|\left(f, h_{1}\right)\right|}{\|f\|}-\frac{\left|\left(g, h_{1}\right)\right|}{\|g\|}\right)^{2}= \begin{cases}\frac{2}{\pi^{2}}\left(\frac{T_{1}(a)}{\|a\|}-\frac{T_{2}(b)}{\pi\|b\|}\right)^{2} & \text { if } \lambda \text { is odd } \\ \frac{2}{\pi^{2}}\left(\frac{T_{1}(a)}{\|a\|}\right)^{2} & \text { if } \lambda \text { is even. }\end{cases}
$$

Hence, the inequality (3.7) can be reduced to

$$
\left|V(a, b)+U_{1}(a, b)\right|^{2} \leq\left\{\pi^{2}\|a\|^{2}\|b\|^{2}\right\}\left(1-r_{1}\right) .
$$

Notice that $U_{1}(b, a)=\overline{U_{1}(a, b)}$ and $V(b, a)=-\overline{V(a, b)}$. If we still select the unit-vector $h_{2}=\sqrt{2 t} / 2 \pi$, then, interchanging $a$ and $b$ in (3.12), we have

$$
\left|-\overline{V(a, b)}+\overline{U_{1}(a, b)}\right|^{2} \leq\left(\pi^{2}\|a\|^{2}\|b\|^{2}\right)\left(1-r_{2}\right),
$$

where $r_{2}$ is defined by

$$
r_{2}=\left(\frac{\left|\left(f, h_{2}\right)\right|}{\|f\|}-\frac{\left|\left(g, h_{2}\right)\right|}{\|g\|}\right)^{2}= \begin{cases}\frac{2}{\pi^{2}}\left(\frac{T_{1}(b)}{\|b\|}-\frac{T_{2}(a)}{\pi\|a\|}\right)^{2} & \text { if } \lambda \text { is odd }, \\ \frac{2}{\pi^{2}}\left(\frac{T_{1}(b)}{\|b\|}\right)^{2} & \text { if } \lambda \text { is even. }\end{cases}
$$

Adding (3.12) and (3.13), then the inequality (3.2) follows after simplifications.

(ii) When $0<\lambda<1$, we firstly consider $h$ in (3.7). We still select unit-vector $h_{1}=\sqrt{2 t} / 2 \pi$. It is easy to deduce that

$$
\begin{aligned}
& \left|\left(f, h_{1}\right)\right|=\sqrt{2}\left|\left(\sum_{m=0}^{\infty} \frac{a_{m}}{u(m)}\right) \cos \lambda \pi-\frac{1}{2 \pi}\left(\sum_{m=0}^{\infty} \frac{a_{m}}{(u(m))^{2}}\right) \sin \lambda \pi\right|, \\
& \left|\left(g, h_{1}\right)\right|=\sqrt{2}\left|\left(\sum_{n=0}^{\infty} \frac{b_{n}}{u(n)}\right) \sin \lambda \pi-\frac{1}{\pi}\left(\sum_{n=0}^{\infty} \frac{b_{n}}{(u(n))^{2}}\right) \sin ^{2} \frac{\lambda \pi}{2}\right| .
\end{aligned}
$$

Since the series $\sum_{n=0}^{\infty} a_{n}$ and $\sum_{n=0}^{\infty} b_{n}$ are absolute convergent, it is justified that the complex numbers $a_{n}$ and $b_{n}$ are replaced, respectively, by $\left|a_{n}\right|$ and $\left|b_{n}\right|$ in the above relations. Let $\left.s_{1}(x)=\left|\left(f, h_{1}\right)\right| /\|f\|\right), s_{2}(x)=\left|\left(g, h_{1}\right)\right| /\|g\|$. By using (3.1), we find $s_{1}(a), s_{2}(b)$. 
Let $R_{1}^{2}=\left(s_{1}(a)-s_{2}(b)\right)^{2}$. We obtain from (3.7)

$$
\begin{aligned}
& \left|V(a, b)+\left(U_{1}(a, b) \cos 2 \lambda \pi-\frac{1}{2 \pi} U_{2}(a, b) \sin 2 \lambda \pi\right)\right|^{2} \\
& \quad \leq\left\{\pi^{2}\|a\|^{2}\|b\|^{2}-\left(\|b\|^{2} r(a)-\|a\|^{2} r(b)\right)-\frac{1}{\pi^{2}} r(a) r(b)\right\}\left(1-R_{1}^{2}\right) .
\end{aligned}
$$

Notice that $U_{1}(b, a)=\overline{U_{1}(a, b)}, U_{2}(b, a)=\overline{U_{2}(a, b)}$, and $V(b, a)=-\overline{V(a, b)}$. If we still select the unit-vector $h_{2}=\sqrt{2 t} / 2 \pi$, then, interchanging $a$ and $b$ in (3.16), we have

$$
\begin{aligned}
& \left|-\overline{V(a, b)}+\left(\overline{U_{1}(a, b)} \cos 2 \lambda \pi-\frac{1}{2 \pi} \overline{U_{2}(a, b)} \sin 2 \lambda \pi\right)\right|^{2} \\
& \quad \leq\left\{\pi^{2}\|a\|^{2}\|b\|^{2}-\left(\|a\|^{2} r(b)-\|b\|^{2} r(a)\right)-\frac{1}{\pi^{2}} r(b) r(a)\right\}\left(1-R_{2}^{2}\right),
\end{aligned}
$$

where $R_{2}^{2}=\left(s_{1}(b)-s_{2}(a)\right)^{2}$. Let $R^{2}=\min \left\{R_{1}^{2}, R_{2}^{2}\right\}$. Adding (3.16) and (3.17), the inequality (3.4) can be gotten after simplifications. In particular, when $1 / 2 \leq \lambda<1$, by Lemma 2.3, we have $r(a) r(b) \geq 0$. The proof of Theorem 3.1 is completed.

Corollary 3.2. Let $\sum_{n=0}^{\infty} a_{n}$ be absolute convergent. Then,

(i) if $\lambda$ is an integer, then

$$
\left|U_{1}(a)\right|^{2}+|V(a)|^{2} \leq\left(\pi^{2}\|a\|^{4}\right)\left(1-R^{2}\right),
$$

where

$$
R^{2}= \begin{cases}\frac{2}{\pi^{2}\|a\|^{2}}\left(T_{1}(a)-\frac{T_{2}(a)}{\pi}\right)^{2} & \text { if } \lambda \text { is odd } \\ \frac{2}{\pi^{2}\|a\|^{2}}\left(T_{1}(a)\right)^{2} & \text { if } \lambda \text { is even }\end{cases}
$$

(ii) if $0<\lambda<1$, then

$$
\left|U_{1}(a) \cos 2 \lambda \pi-\frac{1}{2 \pi} U_{2}(a) \sin 2 \lambda \pi\right|^{2}+|V(a)|^{2} \leq\left\{\pi^{2}\|a\|^{4}-\frac{1}{\pi^{2}} r^{2}(a)\right\}\left(1-R^{2}\right),
$$

where $R^{2}=\left(s_{1}(a)-s_{2}(a)\right)^{2}, s_{i}(x)(i=1,2)$ is defined by (3.1).

In particular, when $u(n)=n+\lambda / 2$, according to (3.2), one obtains a refinement of (1.3) immediately.

Corollary 3.3. If $\lambda=0,1$, then

$$
\left|\sum_{m=1-\lambda}^{\infty} \sum_{n=1-\lambda}^{\infty} \frac{a_{m} \overline{b_{n}}}{m+n+\lambda}\right|^{2}+\left|\sum_{\substack{m=1-\lambda \\ m}}^{\infty} \sum_{\substack{n=1-\lambda \\ m \neq n}}^{\infty} \frac{a_{m} \overline{b_{n}}}{m-n}\right|^{2} \leq\left(\pi^{2} \sum_{n=1-\lambda}^{\infty}\left|a_{n}\right|^{2} \sum_{n=1-\lambda}^{\infty}\left|b_{n}\right|^{2}\right)\left(1-R^{2}\right),
$$


where

$$
R^{2}= \begin{cases}\frac{1}{\pi^{2}}\left\{\left(\frac{T_{1}(a)}{\|a\|}-\frac{T_{2}(b)}{\pi\|b\|}\right)^{2}+\left(\frac{T_{1}(b)}{\|b\|}-\frac{T_{2}(a)}{\pi\|a\|}\right)^{2}\right\} & \text { if } \lambda=1, \\ \frac{1}{\pi^{2}}\left(\left(\frac{T_{1}(a)}{\|a\|}\right)^{2}+\left(\frac{T_{1}(b)}{\|b\|}\right)^{2}\right) & \text { if } \lambda=0\end{cases}
$$

where

$$
T_{k}(x)=\sum_{n=1-\lambda}^{\infty} \frac{\left|x_{n}\right|}{(n+\lambda / 2)^{k}}, \quad k=1,2 .
$$

Corollary 3.4. If $u(n)=n+1 / 4$, then

$$
\left|\left(\sum_{m=0}^{\infty} \sum_{n=0}^{\infty} \frac{a_{m} \bar{b}_{n}}{m+n+1 / 2}\right)\right|^{2}+\left|\sum_{m=0}^{\infty} \sum_{n=0, m \neq n}^{\infty} \frac{a_{m} \bar{b}_{n}}{m-n}\right|^{2} \leq\left\{\pi^{2}\|a\|^{2}\|b\|^{2}-\frac{1}{\pi^{2}} r(a) r(b)\right\}\left(1-R^{2}\right),
$$

where

$$
\begin{aligned}
r(a) r(b)>0, & \quad R^{2}=\min \left\{\left(s_{1}(a)-s_{2}(b)\right)^{2},\left(s_{1}(b)-s_{2}(a)\right)^{2}\right\}, \\
s_{1}(x) & =\frac{\left|-(1 / \sqrt{2} \pi) T_{2}(x)\right|}{\left(\pi^{2}\|x\|^{2}-r(x)\right)^{1 / 2}} \\
s_{2}(x) & =\frac{\sqrt{2}\left|T_{1}(x)-(1 / 2 \pi) T_{2}(x)\right|}{\left(\pi^{2}\|x\|^{2}+r(x)\right)^{1 / 2}}, \\
T_{k}(x) & =\sum_{n=0}^{\infty} \frac{\left|x_{n}\right|}{(n+1 / 4)^{k}}, \quad k=1,2 .
\end{aligned}
$$

Since $\lambda=1 / 2$, it is known from Lemma 2.3 that $r(a) r(b)>0$.

If $r(a) r(b)$ and $R$ in (3.24) are replaced by zero, then the inequality (3.24) can be reduced to

$$
\left|\left(\sum_{m=0}^{\infty} \sum_{n=0}^{\infty} \frac{a_{m} \bar{b}_{n}}{m+n+1 / 2}\right)\right|^{2}+\left|\sum_{m=0}^{\infty} \sum_{n=0, m \neq n}^{\infty} \frac{a_{m} \bar{b}_{n}}{m-n}\right|^{2}<\pi^{2}\|a\|^{2}\|b\|^{2} .
$$

The inequalities (3.24) and (3.26) are refinements of the Hilbert-Ingham inequality

$$
\left|\sum_{m=0}^{\infty} \sum_{n=0}^{\infty} \frac{a_{m} \bar{b}_{n}}{m+n+1 / 2}\right| \leq \pi\|a\|\|b\|
$$

One has yet a new inequality according to Theorem 3.1(ii). 
Theorem 3.5. With the assumptions as Theorem 3.1, if $\lambda=1 / 4$, then

$$
\left|\frac{1}{2 \pi} U_{2}(a, b)\right|^{2}+|V(a, b)|^{2} \leq\left\{\pi^{2}\|a\|^{2}\|b\|^{2}-\frac{1}{\pi^{2}} r(a) r(b)\right\}\left(1-R^{2}\right),
$$

where

$$
\begin{aligned}
R^{2} & =\min \left\{\left(s_{1}(a)-s_{2}(b)\right)^{2},\left(s_{1}(b)-s_{2}(a)\right)^{2}\right\}, \\
s_{1}(x) & =\frac{\left|T_{1}(x)-(1 / 2 \pi) T_{2}(x)\right|}{\left(\pi^{2}\|x\|^{2}-r(x)\right)^{1 / 2}}, \\
s_{2}(x) & =\frac{\left|T_{1}(x)-(1 / 2 \pi) T_{2}(x)(\sqrt{2}-1)\right|}{\left(\pi^{2}\|x\|^{2}+r(x)\right)^{1 / 2}} \\
T_{k}(x) & =\sum_{n=0}^{\infty} \frac{\left|x_{n}\right|}{(n+1 / 8)^{k}}, \quad k=1,2 .
\end{aligned}
$$

\section{Applications to $H_{P}$ function}

Let $f(z)$ be analytic in the unit-disc $|z|<1$. If $f(z)=\sum_{n=0}^{\infty} a_{n} z^{n} \in H_{p}$ with $p>0$, then

$$
\int_{0}^{1}|f(t)|^{p} d t \leq \frac{1}{2} \int_{0}^{2 \pi}\left|f\left(e^{i t}\right)\right|^{p} d t
$$

where the coefficient $1 / 2$ is the best possible. It is called the Fejer-Riesz inequality in $H_{p}$ function [7].

We will give both an extension and a refinement of (4.1) in what follows.

Theorem 4.1. Let $f(z)=\sum_{n=0}^{\infty} a_{n} z^{u(n)-1 / 2} \in H_{p}$, where $p>0$ and $u(n)=Z_{n}+(\lambda / 2)\left(Z_{n} \in Z, \lambda \in\right.$ $N_{0}$ ). If $f(z)$ is analytic in the unit-disc $|z|<1$, then

$$
\left.\left.\left|\int_{0}^{1}\right| f(t)\right|^{p} d t\right|^{2}+\left.\left.\left|\frac{1}{2 \pi} \int_{-\pi}^{\pi} t\right| f\left(-e^{i t}\right)\right|^{p} d t\right|^{2} \leq\left(\frac{1}{2} \int_{0}^{2 \pi}\left|f\left(e^{i t}\right)\right|^{p} d t\right)^{2}\left(1-R^{2}\right),
$$

where $R^{2}>0$.

Proof. At first, we prove the theorem for case $p=2$. Let $f(z)=\sum_{m=0}^{\infty} a_{m} z^{u(m)-1 / 2}$. It is easy to deduce that

$$
\begin{aligned}
\int_{0}^{1}|f(t)|^{2} d t= & \sum_{m=0}^{\infty} \sum_{\substack{u=0 \\
u(m)+u(n) \neq 0}}^{\infty} \frac{a_{m} \overline{a_{n}}}{u(m)+u(n)}=U_{1}(a), \\
& \frac{1}{2 \pi} \int_{0}^{2 \pi}\left|f\left(e^{i t}\right)\right|^{2} d t=\|a\|^{2} .
\end{aligned}
$$


By Lemma 2.4, we have

$$
\left.\left|\frac{1}{2 \pi} \int_{-\pi}^{\pi} t\right| f\left(-e^{i t}\right)\right|^{2} d t|=| \sum_{m=0}^{\infty} \sum_{\substack{n=0 \\ u(m) \neq u(n)}}^{\infty} \frac{a_{m} \overline{a_{n}}}{u(m)-u(n)}|=| V(a) \mid .
$$

Since the series $\sum_{n=0}^{\infty} a_{n}$ is absolutely convergent, it is justified that the complex number $a_{n}$ is replaced by $\left|a_{n}\right|$.

According (3.18), we have

$$
\left.\left.\left|\int_{0}^{1}\right| f(t)\right|^{2} d t\right|^{2}+\left.\left.\left|\frac{1}{2 \pi} \int_{-\pi}^{\pi} t\right| f\left(-e^{i t}\right)\right|^{2} d t\right|^{2} \leq\left(\frac{1}{2} \int_{0}^{2 \pi}\left|f\left(e^{i t}\right)\right|^{2} d t\right)^{2}\left(1-R^{2}\right),
$$

where $R^{2}$ is defined by (3.19). It is easy to deduce that

$$
\begin{aligned}
\frac{2}{\pi^{2}\|a\|^{2}} & =\frac{4}{\pi}\left(\int_{0}^{2 \pi}\left|f\left(e^{i t}\right)\right|^{2} d t\right)^{-1}, \\
T_{1}(a) & =\int_{0}^{1} t^{-1 / 2}|f(t)| d t, \\
T_{2}(a) & =\int_{0}^{1} \frac{d s}{s} \int_{0}^{s} t^{-1 / 2}|f(t)| d t .
\end{aligned}
$$

Because $u(n)=Z_{n}+\lambda / 2 \neq 1 / \pi, T_{1}(a)-T_{2}(a) / \pi \neq 0$. It shows that $R^{2}>0$. Hence, the inequality (4.2) is valid when $p=2$. If $p \neq 2$, then by the Blaschke decomposition theorem, it holds that $f(z)=B(z) G(z)$, where $B(z)$ is Blaschke function and $G(z) \neq 0,|B(z)| \leq 1$ in $|z|<1$ and $\left|B\left(e^{i t}\right)\right|=1$.

Let $F(z)=(G(z))^{p / 2} \in H_{2}$. According to the above result for $p=2$, we have

$$
\begin{aligned}
\left.\int_{0}^{1}|f(t)|^{p} d t\right|^{2}+\left.\left.\left|\frac{1}{2 \pi} \int_{-\pi}^{\pi} t\right| f\left(-e^{i t}\right)\right|^{p} d t\right|^{2} & =\left.\left.\left|\int_{0}^{1}\right| F(t)\right|^{2} d t\right|^{2}+\left.\left.\left|\frac{1}{2 \pi} \int_{-\pi}^{\pi} t\right| F\left(-e^{i t}\right)\right|^{2} d t\right|^{2} \\
& \leq\left\{\frac{1}{2} \int_{0}^{2 \pi}\left|F\left(e^{i t}\right)\right|^{2} d t\right\}^{2}\left(1-R_{F}^{2}\right) \\
& =\left\{\frac{1}{2} \int_{0}^{2 \pi}\left|G\left(e^{i t}\right)\right|^{p} d t\right\}^{2}\left(1-R_{G}^{2}\right) \\
& =\left\{\frac{1}{2} \int_{0}^{2 \pi}\left|f\left(e^{i t}\right)\right|^{p} d t\right\}^{2}\left(1-R^{2}\right) .
\end{aligned}
$$

Based on the case for $p=2$, we have $R_{F}^{2}>0$. Hence, $R^{2}>0$. The proof of Theorem 4.1 is completed.

Let

$$
f(z)=\sum_{m=0}^{\infty} c_{m} z^{m} \in H_{1} .
$$


Then,

$$
\sum_{n=0}^{\infty} \frac{\left|c_{n}\right|}{n+1} \leq \frac{1}{2} \int_{-\pi}^{\pi}\left|f\left(e^{i t}\right)\right| d t
$$

It is called the Hardy inequality in $H_{p}$ function [7].

We will give both an extension and a refinement of (4.9) as follows.

Theorem 4.2. Let $f(z)=\sum_{m=0}^{\infty} c_{m} z^{u(m)}$ be analytic in the unit-disc $|z|<1$, where $u(m)=Z_{m}+\lambda / 2$ (with $Z_{m} \in Z$ and $\lambda \in N_{0}$ ) and $f \in H_{1}$. Then,

$$
\left(\sum_{n=0}^{\infty} \frac{\left|c_{n}\right|}{u(n)}\right)^{2}+\left.\left.\left|\frac{1}{2 \pi} \int_{-\pi}^{\pi} t\right| f\left(-e^{i t}\right)\right|^{2} d t\right|^{2} \leq\left(\frac{1}{2} \int_{-\pi}^{\pi}\left|f\left(e^{i t}\right)\right| d t\right)^{2}\left(1-R^{2}\right),
$$

where

$$
R^{2}= \begin{cases}\frac{4}{\pi}\left(\int_{0}^{1} t^{-1}|f(t)| d t-\frac{1}{\pi} \int_{0}^{1} \frac{d s}{s} \int_{0}^{s} t^{-1}|f(t)| d t\right)^{2}\left(\int_{0}^{2 \pi}\left|f\left(e^{i t}\right)\right| d t\right)^{-1} & \text { if } \lambda \text { is odd } \\ \frac{4}{\pi}\left(\int_{0}^{1} t^{-1}|f(t)| d t\right)^{2}\left(\int_{0}^{2 \pi}\left|f\left(e^{i t}\right)\right| d t\right)^{-1} & \text { if } \lambda \text { is even } .\end{cases}
$$

Proof. By Blaschke decomposition theorem, we have

$$
f(z)=B(z) G(z)=B(z) G^{1 / 2}(z) G^{1 / 2}(z)=f_{1}(z) f_{2}(z),
$$

where $B(z)$ is Blaschke function, $f_{1}, f_{2} \in H_{2}$. Let $f_{1}(z)=B(z) G^{1 / 2}(z)=\sum_{m=0}^{\infty} a_{m} z^{u(m)}, f_{2}(z)=$ $G^{1 / 2}(z)=\sum_{n=0}^{\infty} b_{n} z^{u(n)}$. It is easy to deduce that

$$
\begin{aligned}
\|a\|^{2} & =\|b\|^{2}=\sum_{m=0}^{\infty}\left|a_{m}\right|^{2}=\sum_{n=0}^{\infty}\left|b_{n}\right|^{2}=\frac{1}{2 \pi} \int_{-\pi}^{\pi}\left|G^{1 / 2}\left(e^{i t}\right)\right|^{2} d t \\
& =\frac{1}{2 \pi} \int_{-\pi}^{\pi}\left|B\left(e^{i t}\right) G^{1 / 2}\left(e^{i t}\right)\right|^{2} d t=\frac{1}{2 \pi} \int_{0}^{2 \pi}\left|f\left(e^{i t}\right)\right| d t .
\end{aligned}
$$

Owing to $f(z)=f_{1}(z) f_{2}(z)$, it holds that $\int_{0}^{1} t^{-1}|f(t)| d t=\int_{0}^{1} t^{-1}\left|f_{1}(t)\right|^{2} d t$. It is easy to deduce that

$$
\sum_{n=0}^{\infty} \frac{\left|c_{n}\right|}{u(n)} \leq \sum_{n=0}^{\infty} \sum_{r+s=n} \frac{\left|a_{r}\right|\left|a_{s}\right|}{u(r)+u(s)}=\sum_{m=0}^{\infty} \sum_{n=0}^{\infty} \frac{\left|a_{m}\right|\left|a_{n}\right|}{u(m)+u(n)} .
$$

By Lemma 2.4, we find that

$$
\left|\frac{1}{2 \pi} \int_{-\pi}^{\pi} t\right| f\left(-e^{i t}\right)|d t|=\left.\left|\frac{1}{2 \pi} \int_{-\pi}^{\pi} t\right| f_{1}\left(-e^{i t}\right)\right|^{2} d t|=| \sum_{m=0}^{\infty} \sum_{\substack{n=0 \\ u(m) \neq u(n)}}^{\infty} \frac{\left|a_{m}\right|\left|a_{n}\right|}{u(m)-u(n)} \mid .
$$


It follows from (4.13), (4.14), (4.15), and Corollary 3.2 that

$$
\left(\sum_{n=0}^{\infty} \frac{\left|c_{n}\right|}{u(n)}\right)^{2}+\left|\frac{1}{2 \pi} \int_{-\pi}^{\pi} t\right| f\left(-e^{i t}\right)|d t|^{2} \leq\left(\frac{1}{2} \int_{0}^{2 \pi}\left|f\left(e^{i t}\right)\right| d t\right)^{2}\left(1-R^{2}\right),
$$

where $R^{2}$ is defined by (3.19). It is easy to deduce that

$$
\begin{aligned}
\frac{2}{\pi^{2}\|a\|^{2}} & =\frac{4}{\pi}\left(\int_{0}^{2 \pi}\left|f\left(e^{i t}\right)\right|^{2} d t\right)^{-1}, \\
T_{1}(a) & =\int_{0}^{1} t^{-1}\left|f_{1}(t)\right|^{2} d t=\int_{0}^{1} t^{-1}|f(t)| d t \\
T_{2}(a) & =\int_{0}^{1} \frac{d s}{s} \int_{0}^{s} t^{-1}\left|f_{1}(t)\right|^{2} d t=\int_{0}^{1} \frac{d s}{s} \int_{0}^{s} t^{-1}|f(t)| d t .
\end{aligned}
$$

These show that the inequality (4.10) is valid.

\section{Acknowledgment}

The research is supported by the Scientific Research Fund of Hunan Provincial Education Department (no. 06C657).

\section{References}

[1] K. Hu, "On Hilbert's inequality," Chinese Annals of Mathematics. Series B, vol. 13, no. 1, pp. 35-39, 1992.

[2] F. Zeng, M. Gao, and L. He, "On the symmetric Hilbert's inequality and its applications," Mathematical Inequalities \& Applications, vol. 6, no. 1, pp. 45-53, 2003.

[3] J. C. Kuang, Applied Inequalities, Shandong Science and Technology Press, Jinan, China, 3rd edition, 2004.

[4] M. Krnić, M. Gao, J. Pečarić, and X. Gao, "On the best constant in Hilbert's inequality," Mathematical Inequalities \& Applications, vol. 8, no. 2, pp. 317-329, 2005.

[5] M. Gao, "On Heisenberg's inequality," Journal of Mathematical Analysis and Applications, vol. 234, no. 2, pp. 727-734, 1999.

[6] M. Gao, L. Tan, and L. Debnath, "Some improvements on Hilbert's integral inequality," Journal of Mathematical Analysis and Applications, vol. 229, no. 2, pp. 682-689, 1999.

[7] P. L. Duren, Theory of $H_{p}$ Spaces, Pure and Applied Mathematics, Academic Press, New York, NY, USA, 1970. 


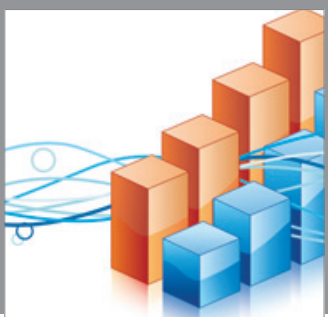

Advances in

Operations Research

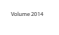

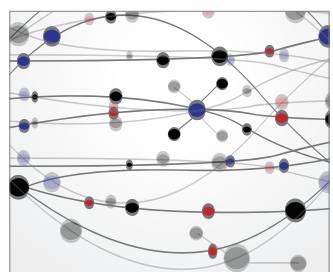

\section{The Scientific} World Journal
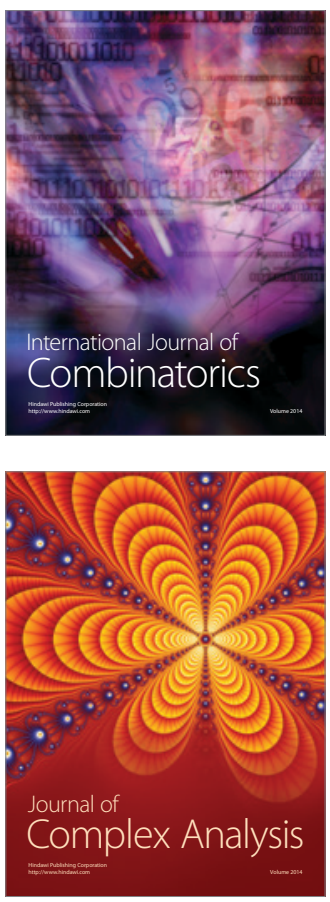

International Journal of

Mathematics and

Mathematical

Sciences
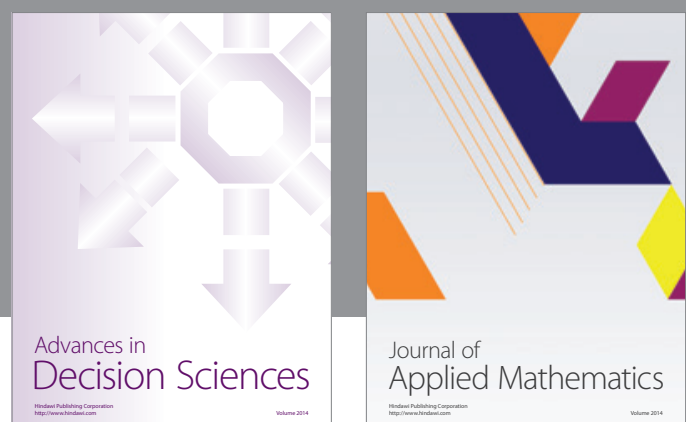

Journal of

Applied Mathematics
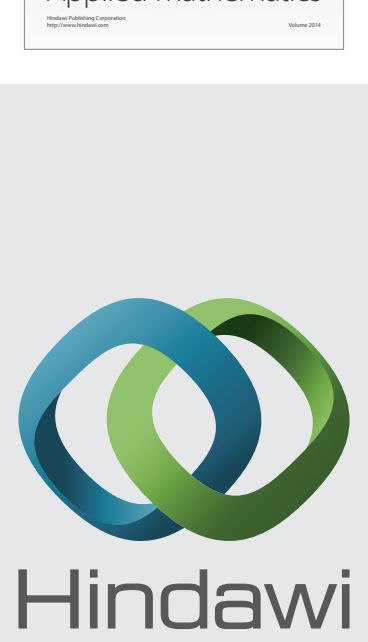

Submit your manuscripts at http://www.hindawi.com
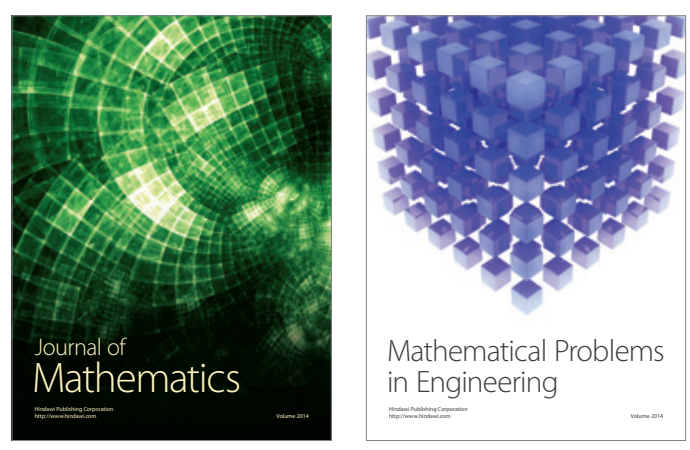

Mathematical Problems in Engineering
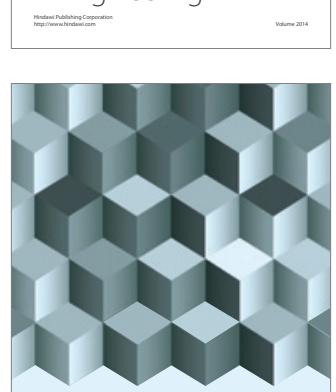

Journal of

Function Spaces
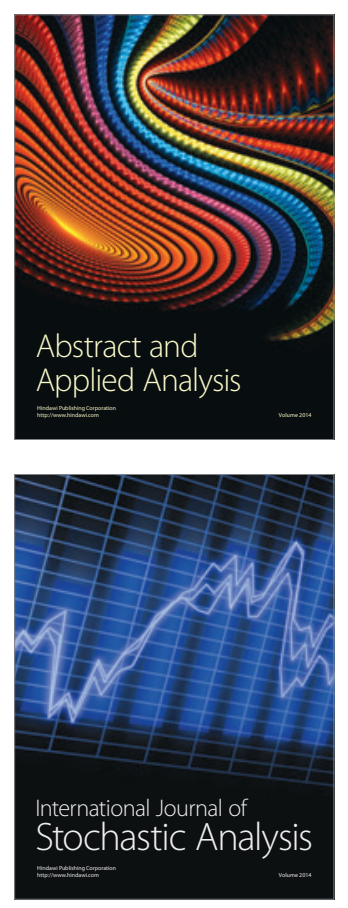

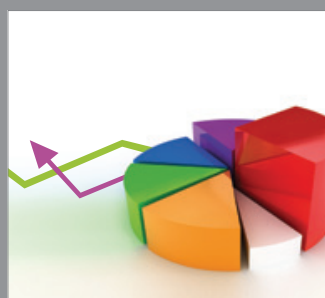

ournal of

Probability and Statistics

Promensencen
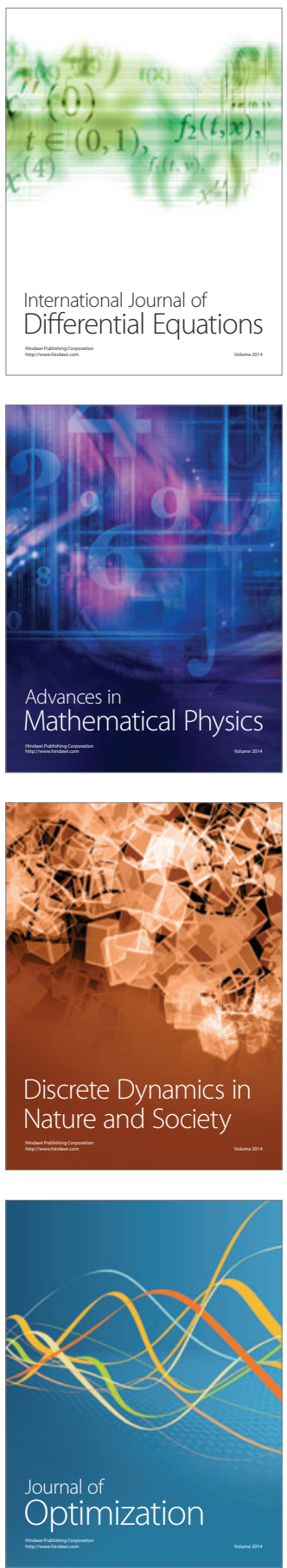